\title{
Interpretive nutrient profiling algorithms are a limited tool for assessing the healthiness of countries' packaged food and beverage supplies, and the conclusions are not substantiated by the data
}

To the Editor,

We read with interest the article by Dunford and colleagues ${ }^{1}$ comparing the 'healthiness' of packaged foods and beverages across twelve countries using nutrient profiling algorithms developed for Australia's interpretive front of pack nutrition labelling system, the Health Star Rating (HSR). Access to a nutritious, well balanced diet is an important determinant of health, and, with the rise in packaged food consumption in recent decades, monitoring the availability and nutritional quality of these products is timely.

The objective of Dunford et al's paper was to use the HSR to examine the 'healthiness' of national packaged food and beverage supplies. With the recommendations that the study's methodology be incorporated into an annual survey comparing countries' food supplies, it is important to consider the methodology used and whether the conclusions are warranted.

The veracity of the HSR as the measure of healthiness is not questioned in the paper nor is it justified. As such, the conclusions of the paper rest on the assumption that the HSR is an accurate measure of the healthiness of different country's food supplies. While the authors posit that the HSR is "well suited to a comparative analysis of packaged foods and beverages available in different countries" we contend that the HSR provides a limited indication of the healthiness of foods for a number of reasons.

Firstly, the HSR algorithm does not consider the full range of essential nutrients as it is based on just four so-called "nutrients of concern" ("the densities of energy, sodium, total sugars and saturated fat), and some "positive nutrients" ("the densities of fruit and vegetable content, protein, fibre and in some cases calcium"). This narrow view means that the algorithm is unable to take into account nutritional adequacy implications in its use.

Second, it is a nutrition science truism that food and health relationships are predicated on more than the sum of the nutrients a food contains. This is because of synergies between nutrients in a food, and the interaction of nutrients with the food matrix. ${ }^{2}$ Yet, the HSR does not consider the combination of nutrients within a food matrix and the overall effect of the particular food on health. Using just isolated nutrients to assess a foods' healthiness is problematic. For example, recent analyses indicate a substantial number of foods associated with adverse effects on health, such as ultra-processed foods ${ }^{3}$ and foods the Australian Dietary Guidelines advise should only be consumed "sometimes and in small

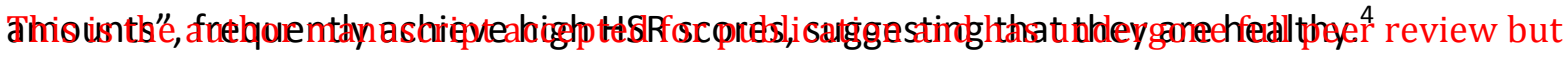
has not been through the copyediting, typesetting pagination and proofreading process, which Third, the HSR system was specifically designed and calculated to rank the foods available within the Australian and New Zealand food supply according to nutrient content, and so is as doi: 10.1111/obr.12957 
country specific. Countries with a different food supply may need to adjust the cut-off points to assign the HSR scores to reflect the different ranges of foods available.

Fourth, the main result included in the abstract was a mean HSR score across all foods and beverages, yet the HSR was not designed to compare different categories of foods. Rather, its intended use is to compare similar products (e.g. different types of breakfast cereal rather than pizza versus breakfast cereal).

The final sentence of the conclusion to Dunford and colleagues' paper reads: "Nutrient profiling is an important tool that can help define and monitor policy actions to improve the healthfulness of products in the market place and reduce population risks of obesity, and non-communicable disease risks". In light of our points above, we do not believe this is a reasonable conclusion.

Another of the conclusions of the paper is that "packaged foods and beverages are consistently less healthy in countries such as China and India compared with higher income countries", with "Canada, UK, Australia and UK" given as examples of "high-income countries" (page 6). However, one in five products in the datasets were excluded due to missing data and $11 \%$ of products required imputation of one or more nutrients, yet no information is included in the paper to assess if these exclusions and imputations were evenly spread across countries and categories of packaged food and drink products.

Also, no information is presented about how representative the data collected from India (2014-17) and China (2015-18) are of the packaged foods and beverages available in these geographically and socio-economically diverse countries. Relying on ownership of a smart phone for crowd-sourcing of data may have introduced both age and socio-economic bias, as at that time, smart phones tended to be more likely to be owned by younger rather than older adults and by those with higher rather than lower socio-economic status. In 2015 only $43 \%$ of Chinese 5 and $18 \%$ of Indian adults owned a smart phone. ${ }^{6}$ While $66 \%$ of adults owned a smart phone in the UK, this comprised of $90 \%$ of $16-24$ year olds, but only $18 \%$ of adults over the age of 65 years. ${ }^{7}$

According to Table 1 in the paper, products from India represented just $2.1 \%$ of the total number of packaged foods and beverages analysed in the paper. This sample of 8,193 foods is less than one eighth the size of the sample from the UK. The smaller the sample, the lower the chance it is representative of the entire country's packaged food and beverage supply.

With the absence of statistical tests, it is unknown if the observed differences between countries were due to chance, sampling differences, or are likely to represent a true difference. The differences were very small - the mean HSR for foods and beverages for the three high income countries specified (UK, Canada and Australia) was 2.70. This is just 0.5 Health Stars higher than in India and 0.4 Health Stars higher than in China. As there is, to our knowledge, no evidence directly linking Health Stars to diet quality or health outcomes, the relevance of such small differences is unknown. Moreover, improved health outcomes are related to reductions in free sugars ${ }^{8}$ (rather than total sugars - which is an input into the HSR). Therefore, the conclusion that "packaged foods and beverages are consistently less

This article is protected by copyright. All rights reserved. 
healthy in countries such as China and India compared with higher income countries" is not demonstrated by the results presented.

Overall, we suggest that Dunford and colleagues' conclusions are inadequately substantiated by the data. This is because of the assumption that the HSR nutrient profiling algorithms provide a reliable tool to assess a food's healthiness, and due to a number of methodological limitations as outlined. As a result the conclusions of this paper, are, in our view, misleading.

Yours sincerely

Mark Lawrence

Anita Lawrence

Christina Pollard

Claire Pulker

Julie Woods

Jennifer Lacy-Nichols

Bronwyn Ashton

Rosemary Stanton

\section{References}

1. Dunford EK, Ni Mhurchu C, Huang I, Vandevijvere S, Swinburn S, Pravst I. A comparison of the healthiness of packaged foods and beverages from 12 countries using the Health Star Rating nutrient profiling system, 2013-2018. Obes Rev, 2019;19. https://doi.org/10.1111/obr.12879

2. Jacobs DR, Tapsell LC. Food, not nutrients, is the fundamental unit in nutrition. Nutr Rev, 2007; 65(10):439-450. https://doi.org/10.1111/i.1753-4887.2007.tb00269.x

3. Dickie S, Woods JL, Lawrence MA. Analysing the use of the Australian Health Star Rating system by level of food processing. Int J Beh Nutr Phys Act, 2018;15(1):128. https://doi.org/10.1186/s12966-018-0760-7

4. Lawrence MA, Dickie S, Woods JL. Do nutrient-based Front-of-Pack Labelling schemes support or undermine Food-Based Dietary Guideline recommendations? Lessons from the Australian Health Star Rating system. Nutrients, 2018;10(1). https://doi.org/10.3390/nu10010032 
5. Statista. Smartphone penetration rate in China from 2015 to 2023, https://www.statista.com/statistics/321482/smartphone-user-penetration-in-china/ (Accessed September 2019).

6. Statista. Share of mobile phone users that use a smartphone in India from 2014 to 2022, https://www.statista.com/statistics/257048/smartphone-user-penetration-inindia/(Accessed September 2019).

7. Ofcom. The Communications Market Research; 2015, https://www.ofcom.org.uk/_data/assets/pdf_file/0022/20668/cmr_uk_2015.pdf (Accessed September 2019).

8. World Health Organization. Guideline: Sugars intake for adults and children. Geneva: World Health Organization; 2015.

This article is protected by copyright. All rights reserved. 


\section{University Library}

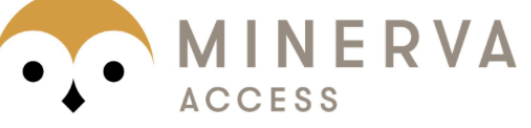

A gateway to Melbourne's research publications

Minerva Access is the Institutional Repository of The University of Melbourne

Author/s:

Lawrence, M;Lawrence, A;Stanton, R;Pollard, C;Pulker, C;Woods, J;Ashton, B

Title:

Interpretive nutrient profiling algorithms are a limited tool for assessing the healthiness of countries' packaged food and beverage supplies, and the conclusions are not substantiated by the data

Date:

2019-12-19

Citation:

Lawrence, M., Lawrence, A., Stanton, R., Pollard, C., Pulker, C., Woods, J. \& Ashton, B. (2019). Interpretive nutrient profiling algorithms are a limited tool for assessing the healthiness of countries' packaged food and beverage supplies, and the conclusions are not substantiated by the data. OBESITY REVIEWS, 21 (2), https://doi.org/10.1111/obr.12957.

Persistent Link:

http://hdl.handle.net/11343/286763 